\title{
La Actividad Científica de la Asociación Española de Urología recogida en las publicaciones periódicas
}

\author{
M. Pérez Albacete \\ Servicio de Urología. Hospital Universitario Virgen de la Arrixaca. Murcia. \\ Actas Urol Esp 2006; 30 (3): 243-253
}

\section{RESUMEN}

\section{LA ACTIVIDAD CIENTÍFICA DE LA ASOCIACIÓN ESPAÑOLA DE UROLOGÍA RECOGIDA EN LAS PUBLICACIONES PERIÓDICAS}

Introducción y objetivos: Desde su constitución la Asociación Española de Urología establece en sus estatutos la necesidad de contar con un órgano propio de difusión; a lo largo de su existencia varias publicaciones han realizado esta función, de modo oficioso unas y oficial otras. Se pretende localizar las actividades efectuadas y el trabajo llevado a cabo por sus miembros, conocer qué revistas médicas las han recogido y cuales han llevado oficialmente su representación, con la finalidad de valorar la importancia que ha tenido en el desarrollo de la Urología española.

Métodos: Revisamos las diferentes publicaciones periódicas con contenido médico editadas en Madrid desde 1911, en las podríamos localizar trabajos o noticias sobre la Asociación y la labor efectuada por sus componentes.

Resultados: Encontramos datos en diez publicaciones periódicas distintas, cinco contemplan el contenido de los Congresos Nacionales y cinco recogen las actividades periódicas; su secretario general ha dispuesto durante este periodo, de otros cinco medios de comunicación para informar a los asociados y dos semanarios médicos notifican periodísticamente de la mayoría de los actos en la primera mitad del siglo XX; por último cuatro revistas diferentes han llevado el título de órgano oficial.

Conclusiones: Encontramos que no existen lagunas de tiempo para recoger las actividades realizadas por la Asociación Española de Urología en sus noventa y cinco años de historia, su estudio nos permite obtener una completa valoración y establecer la importancia que ha tenido en el desarrollo de la Urología española.

Palabras Clave: Historia de la AEU. Publicaciones urológicas. Historia de la Urología.

\section{ABSTRACT}

\section{SCIENTIFIC ACTIVITIES OF THE SPANISH UROLOGY ASSOCIATION GHATED IN THE PERIODIC PUBLITATIONS}

Introduction and objetive: From the constitution of Urology Spanish Association establishes in its bylaws the need to possess an own organ of diffusion; along its existence several publications have realized this function, in a semiofficial way some and official others. One tries to locate the realized activities and the work led to end by its members, to know what medical magazines they have gathered and which have taken officially its representation, with the purpose of valuing the importance that has had in the development of the Spanish Urology.

Methods: We check the different periodic publications with medical content edited in Madrid from 1911, in we might locate works or news on the Association and the labor effected by its components.

Results: We find information in ten periodic different publications, five contemplate the content of the National Congresses and five gather the periodic activities; its secretary general has had during this period, of other five mass media to inform the partners and two medical weeklies notify journalistically of the majority of the acts in the first half of the XXth century; in sum up four different magazines have taken the title of official organ.

Conclusions: We think that lagoons of time do not exist to gather the activities realized by Urology Spanish Association in its ninety five years of history, its study allows us to obtain a complete valuation and to establish the importance that has had in the development of the Spanish Urology.

Keywords: History AEU. Publications urologyc. History Urology. 
$\mathrm{P}$ ropugnó siempre la Asociación Española de Urología (AEU) desde su constitución disponer de un órgano oficial propio de difusión como revista especializada en donde quedaran reflejadas sus actividades científicas y sociales, lo que, prácticamente, $\mathrm{y}$ tras muchas vicisitudes con intentos malogrados, no se consiguió tener definitivamente hasta la aparición de Actas Urológicas Españolas, sesenta y cinco años después. No obstante, el quehacer que ha desarrollado la Asociación, en sus noventa y cinco años de existencia, queda recogido puntualmente en varias de las publicaciones periódicas y semanarios médicos editados en las diferentes épocas y que, unos de un modo oficial y otros como noticia, recababan y transmitían la información además de las notas remitidas por la secretaría de la Asociación. La recopilación de este testimonio nos permite dar a conocer y valorar la contribución que la $\mathrm{AEU}$ ha desempeñado en la evolución $\mathrm{y}$ en el progreso de la especialidad en nuestro país.

\section{MÉTODOS}

Analizamos el reglamento constitucional de la AEU, aprobado en Madrid en enero de 1911, y repasamos las diferentes modificaciones establecidas a lo largo de los años en las Asambleas Generales celebradas en sus Congresos $^{1}$, asî como las diversas disposiciones dictadas por las Juntas Directivas recogidas en sus actas. Revisamos, siguiendo a Insausti Cordón ${ }^{2}$ y a Maganto Pavón ${ }^{3}$, todas cuantas publicaciones periódicas de contenido quirúrgico, dermatológico y urológico así como aquellas otras de carácter médico general susceptibles de recoger tanto las actividades de la AEU como el trabajo realizado por sus miembros y, también, los semanarios de noticias médicas de la primera mitad del siglo XX que se editaron principalmente en Madrid desde 1911, dado que la labor de la Asociación tuvo casi exclusivamente lugar en la capital hasta 1955.

\section{RESULTADOS}

El reglamento constitucional de la AEU de enero de $1911^{4}$, en su artículo 20 , contempla ya que los trabajos presentados en las Sesiones Científicas de la Asociación, Temas oficiales,
Comunicaciones y Discusiones serán publicados en la forma que acuerde la Junta Directiva, y en la primera modificación y ampliación de los estatutos, aprobada en Madrid, en junta general extraordinaria el 21 de octubre y recogida el 19 de diciembre de $1914^{5}$, en el artículo 15 se señala cuando los fondos de la Asociación lo permitan se publicará un Boletín con los trabajos científicos de las sesiones, cuyas condiciones de publicación se fijarán oportunamente por la Junta General.

En enero de 1924 la Revista Española de Cirugía y Urología fue la primera publicación que ostentó el título de órgano de la Asociación Española de Urología, que mantuvo por espacio de diez años, aunque no hemos podido localizar la disposición oficial que así lo definió. En el reglamento aprobado el 7 de julio de 1932, en el transcurso del III Congreso Hispano Portugués celebrado en Coimbra ${ }^{6}$, el artículo 15 define:

"La Asociación Española de Urología publicará una Revista con el titulo: Revista Española de Urología, que será el órgano oficial de aquélla. La dirección de esta Revista estará encomendada a la Junta Directiva de la Asociación, la cual designará dos de sus miembros como Secretarios de redacción encargados de realizar todos los trabajos referentes a la organización y publicación de la Revista. Ésta se publicará trimestralmente, por lo menos, $y$, cada cuatro números formarán un volumen anual".

Tras la Guerra Civil se reorganizó la AEU durante la celebración de las IV Jornadas Médicas Españolas, en Sevilla, en 1945; el cuatro de mayo se comunicó oficialmente su reconstitución por el elegido presidente Dr. D. Pedro Cifuentes Díaz (1880-1960) ${ }^{7}$; el día cuatro de julio tiene lugar en Madrid la primera reunión y en ella se da lectura al reglamento de 1935 y se propone la reforma de muchos de sus artículos aunque en el libro de actas del V Congreso Hispano Portugués de Urología ${ }^{8}$, publicado en 1947, aparece como vigente el de 1935, sin ninguna modificación; que conozcamos no queda constancia escrita alguna de variación hasta el aprobado en Madrid el seis de junio de $1964^{9}$ en el cual, en el artículo $2^{\circ}$, se dictamina que la revista Archivos Españoles de Urología será el órgano Oficial de la Asociación que refleje las actividades científicas de la misma, con ello se le da 
reconocimiento oficial a la labor que la publicación venía realizando desde sus comienzos al recoger gran parte de las sesiones y de las reuniones de la Asociación y se ratifica el acuerdo de la Junta Directiva tomado en sesión en 1947.

En la Asamblea General celebrada en Málaga el 5 de junio de 1968, en el curso del XXXIII Congreso Nacional, se acuerda la publicación de las Actas de la Asociación Española de Urología con las ponencias al congreso y con las comunicaciones libres ${ }^{10}$. En la Junta General Extraordinaria, desarrollada en Oporto, con ocasión del VIII Congreso Luso-Español, el 25 de junio de $1969^{11}$, sufren un nuevo cambio los estatutos y en el artículo $2^{\circ}$ se recoge que para el desarrollo de su labor científica la revista Actas de la Asociación Española de Urología será Órgano Oficial de la Asociación que refleje las actividades científicas de la misma.

En Granada, en el transcurso de la Asamblea General Ordinaria del XL Congreso, el 12 de junio de 1975, se tomó por unanimidad el acuerdo de facultar a la Junta Directiva para realizar las gestiones necesarias para cumplimentar el artículo $2^{-}$ del reglamento; en el XLI Congreso Nacional de León, el 6 de julio de 1976, fue aprobada por unanimidad la puesta en marcha de la revista Actas Urológicas Españolas así como el nombramiento del Dr. D. Carlos Alférez Villalobos como su director y en la reunión de la Junta Directiva de 12 de noviembre se aprueban los estatutos que han de regir la revista ${ }^{12}$.

En el reglamento ratificado en el LXIII Congreso de Cádiz, en junio de $1998^{13}$, se matiza en el artículo $2^{\circ}$, que la revista Actas Urológicas Españolas será el Órgano Oficial de difusión de la Asociación y reflejará las actividades de la misma. Asímismo, y para adaptarse a los cambios tecnológicos que en el avance científico se vayan produciendo, la Asociación establecerá las vías adecuadas, artículo que, desarrollado en el título V, se mantiene sin alteración hasta su última revisión de junio de 2004.

RELACIÓN DE LAS PUBLICACIONES QUE RECOGEN LA ACTIVIDAD DE LA AEU

Actas de los Congresos Nacionales de la Asociación Española de Urologia. Temas oficiales, comunicaciones y discusiones ${ }^{14}$ (Fig. 1).

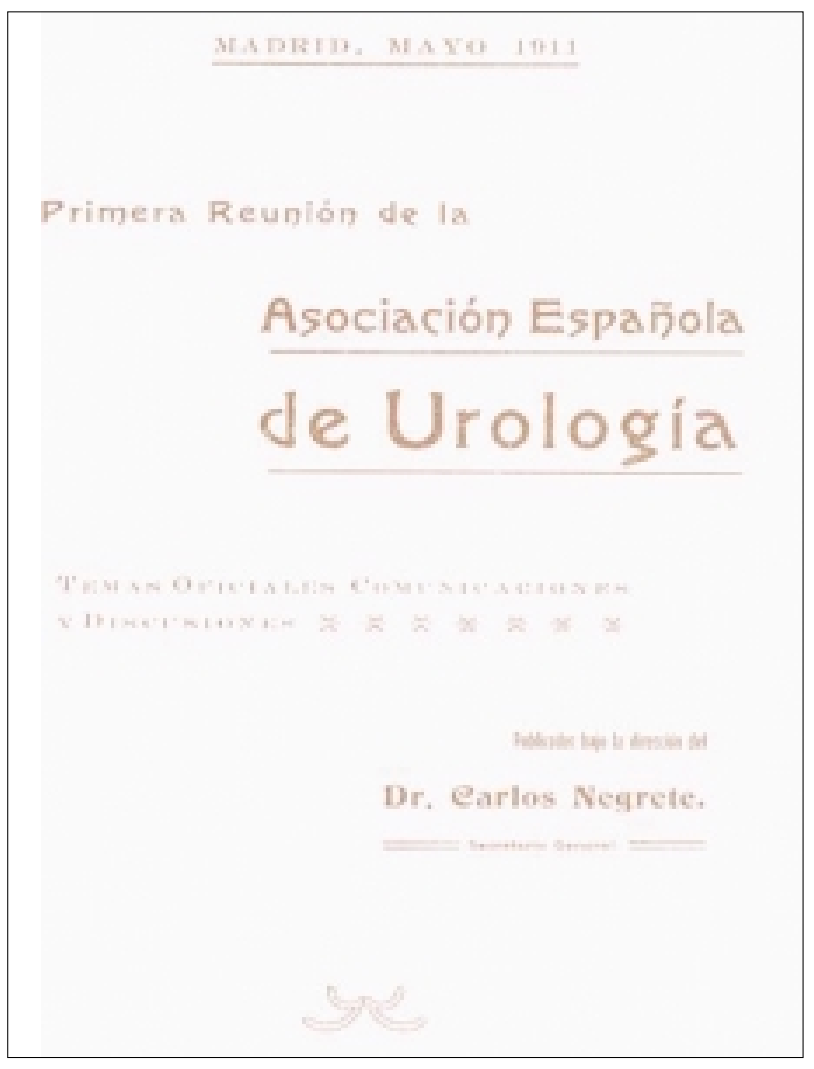

FIGURA 1. Actas de los Congresos Nacionales de la Asociación Española de Urología. Temas Oficiales, comunicaciones $y$ discusiones.

Tras la celebración de la primera Reunión Científica de la Asociación, en Madrid, el 16 de mayo de 1911, a la que posteriormente se consideró como su primer congreso, el secretario general, Dr. D. Carlos Negrete de los Reyes (1885-1932), publica, de acuerdo con los estatutos, el contenido de los temas oficiales presentados así como el de las comunicaciones y discusiones efectuadas, recogidos en formato de libro; esta modalidad de difusión de los textos de los Congresos Nacionales se mantuvo, dirigido por los sucesivos secretarios generales de la AEU, hasta 1946, con lo que se editaron las actividades de los primeros trece congresos en Madrid, excepto dos de los Hispano-Lusos, el I de 1925 y el III de 1932, que lo fue en Lisboa por el secretario de la Asociación Portuguesa.

Revista Española de Urología y Dermatologia ${ }^{15}$. La Revista Española de Sifilografia y Dermatología, que inició su andadura en 1899, en Madrid, dirigida por el dermatólogo Dr. D. Luis del Portillo, en 1914, modificó su nombre por el de 
Revista Española de Urología y Dermatología y, si bien existe una relación entre este cambio y la constitución de la AEU, no consta que fuese oficialmente su órgano representativo o informativo; la Dirección de la publicación justifica el cambio con la indicación de que se ocupará de las enfermedades de los órganos genitourinarios y de las enfermedades de la piel. Mensualmente aparece reflejada entre sus páginas la actividad periódica de la Asociación desde la primera reunión mensual en marzo de 1915 y también artículos urológicos varios junto con los presentados a los Congresos de la especialidad, además de trabajos de dermatología y de venereología, hasta 1924 en que se apareció la Revista Española de Urología; su edición se mantuvo hasta 1927 (Fig. 2).

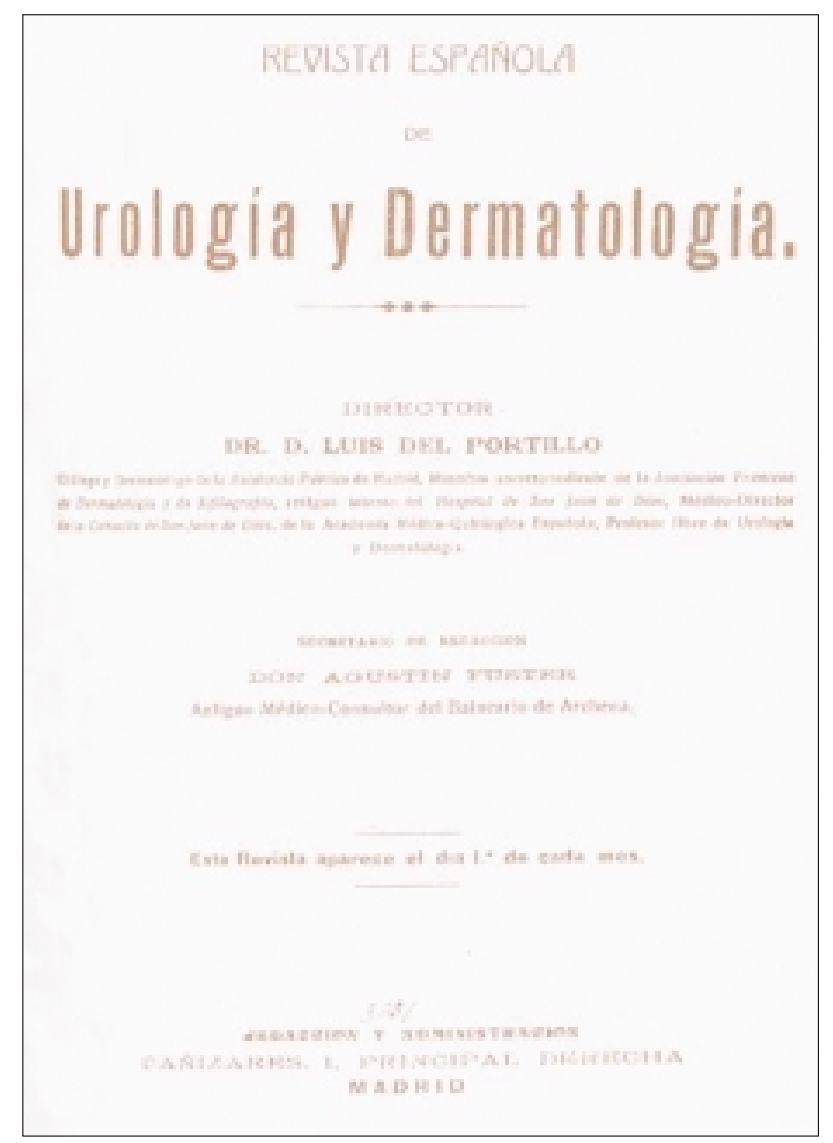

FIGURA 2. Revista Española de Urología y Dermatologia.

Revista Española de Cirugia y Urologia ${ }^{16}$. Fue la primera publicación que ostentó el título oficial de órgano de la Asociación Española de Urología por un espacio de casi diez años, desde enero de 1924 hasta mayo-junio de 1933, en que, por la aparición de la Revista Española de Urología, dejó de representarla, aunque continuó editándose hasta 1935 con el mismo nombre. Fundada en Madrid por los cirujanos Drs. D. Fidel Pagés y D. Tomás R. de Mata, inició su andadura en 1919 con el título de Revista Española de Cirugía y desde siempre mantuvo una estrecha relación con los urólogos, que escribieron un gran número de artículos en sus páginas en las que recoge puntualmente la mayoría de las aportaciones a los congresos de la AEU; en el comité de redacción, ya desde su inicio, encontramos como colaboradores a D. José María Bartrina Thomás (1887-1950), D. Luis González Bravo (1855-1929) y D. Isidro Sánchez Covisa (1879-1944), a quienes, durante su etapa urológica, se añaden los de D. Pedro Cifuentes Díaz, D. Leonardo de la Peña Díaz (1875-1957) y D. Fernando Miraved Blanco (1880-1939), además de incluir los trabajos remitidos por todos los cirujanos y urólogos hispano-americanos lo que, como indica Maganto Pavón, es el primer antecedente escrito para la integración congresual de las Sociedades Españolas y Americanas de Urología (Fig. 3).

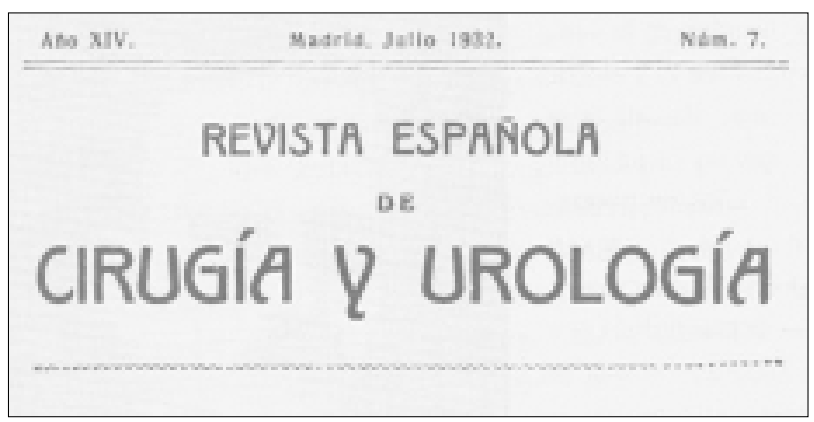

FIGURA 3. Revista Española de Cirugia y Urología.

Boletin de la Asociación Española de Uro$\operatorname{logia}^{17}$. De aparición únicamente en el curso 1923-24, estaba incluido, con formato independiente, en el interior de la Revista Española de Cirugía y Urología. Creado con la intención de dar una información puntual de las actividades, en él se transcriben las actas de las sesiones científicas reglamentarias de la Asociación celebradas mensualmente, así como el título de las comunicaciones presentadas en ellas; en el resto de los años de edición de la revista, notifica entre sus páginas de las sesiones realizadas (Fig. 4). 


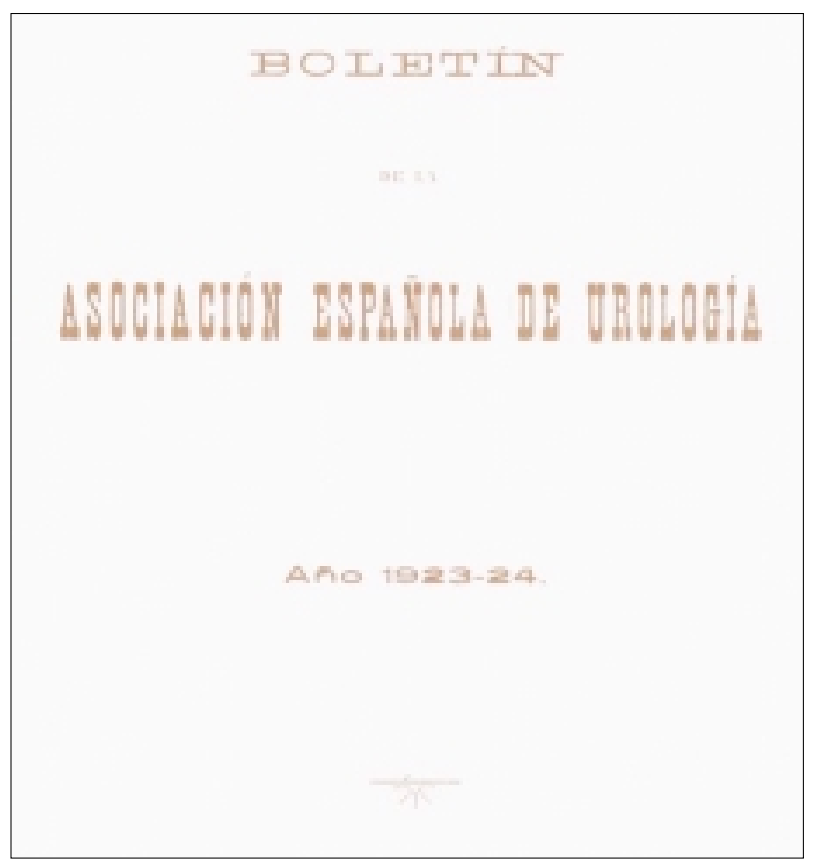

FIGURA 4. Boletin de la Asociación Española de Urologia.

Revista Española de Urología ${ }^{18}$. Órgano Oficial de la Asociación Española de Urología. Con ella se cumplen los anhelos de la Asociación de crear una publicación propia por vez primera desde su constitución; el primer número lleva la fecha de julio-septiembre de 1933 y con periodicidad trimestral, tuvo una vida de sólo tres años ya que la contienda civil desgraciadamente la hizo desaparecer en 1936. De la dirección de la revista se ocupaba la Junta Directiva, encabezada por su presidente, Dr. D. Isidro Sánchez Covisa, quien encargó a los Drs. D. Victoriano Molina y D. Agustín Hidalgo Fernández Cano (1903-1975) actuar como secretarios de redacción responsables de su organización y como colaboradores todos los urólogos españoles $y$ extranjeros que remitan trabajos, la aportación fue fructífera y se incrementó tanto en cantidad como en calidad en cada número; en sus páginas se recogen los actos de las sesiones científicas mensuales celebradas en Madrid (Fig. 5).

Archivos Españoles de Urologia ${ }^{19}$. Tras la Guerra Civil y dado el desolador panorama científico y el vacío de publicaciones urológicas el Dr. D. Enrique Pérez Castro (1908-1980) logra crear, con el apoyo y la colaboración de los Drs. D. Antonio Puigvert Gorro (1905-1987) y D. Luis

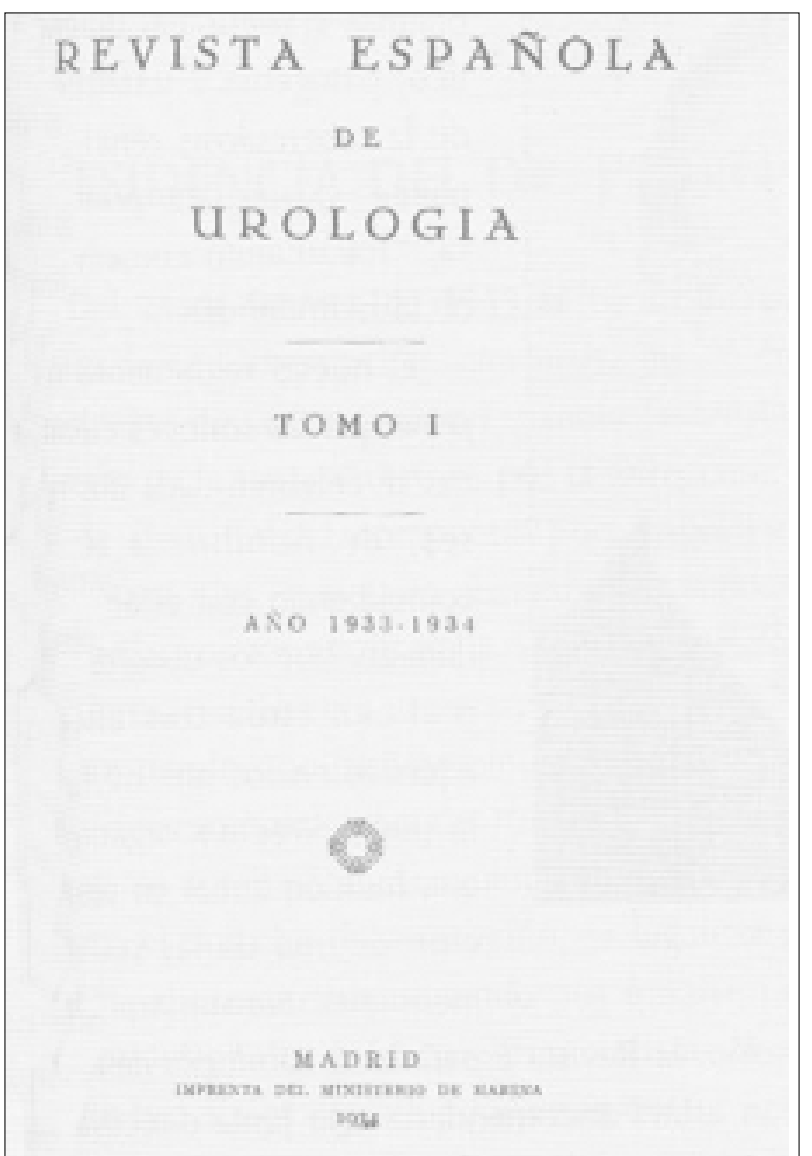

FIGURA 5. Revista Española de Urología.

Cifuentes Delatte (1907-2005), y bajo su dirección, la revista que con el tiempo ha llegado a ser la decana de las urológicas españolas; de aparición en julio de 1944, con periodicidad trimestral al principio y mensual más adelante, se encuentran entre sus páginas el trabajo de todos los urólogos españoles, ya que el deseo de sus promotores fue la de disponer de un medio periodístico en el que quedasen archivados absolutamente todos los trabajos de Urología que se publicasen en España, gracias a esta faceta recopiladora se conserva la producción científica urológica española desde 1944 (Fig. 6). En un apartado de noticias iba informando de las escasas actividades que preconizaba la Asociación durante esos años, pese a todo, hasta 1964 no fue reconocida oficialmente como el órgano oficial de la Asociación, según acuerdo adoptado por junta general extraordinaria celebrada en Madrid el seis de junio, posición en la que se mantuvo hasta la aparición de Actas Urológicas 


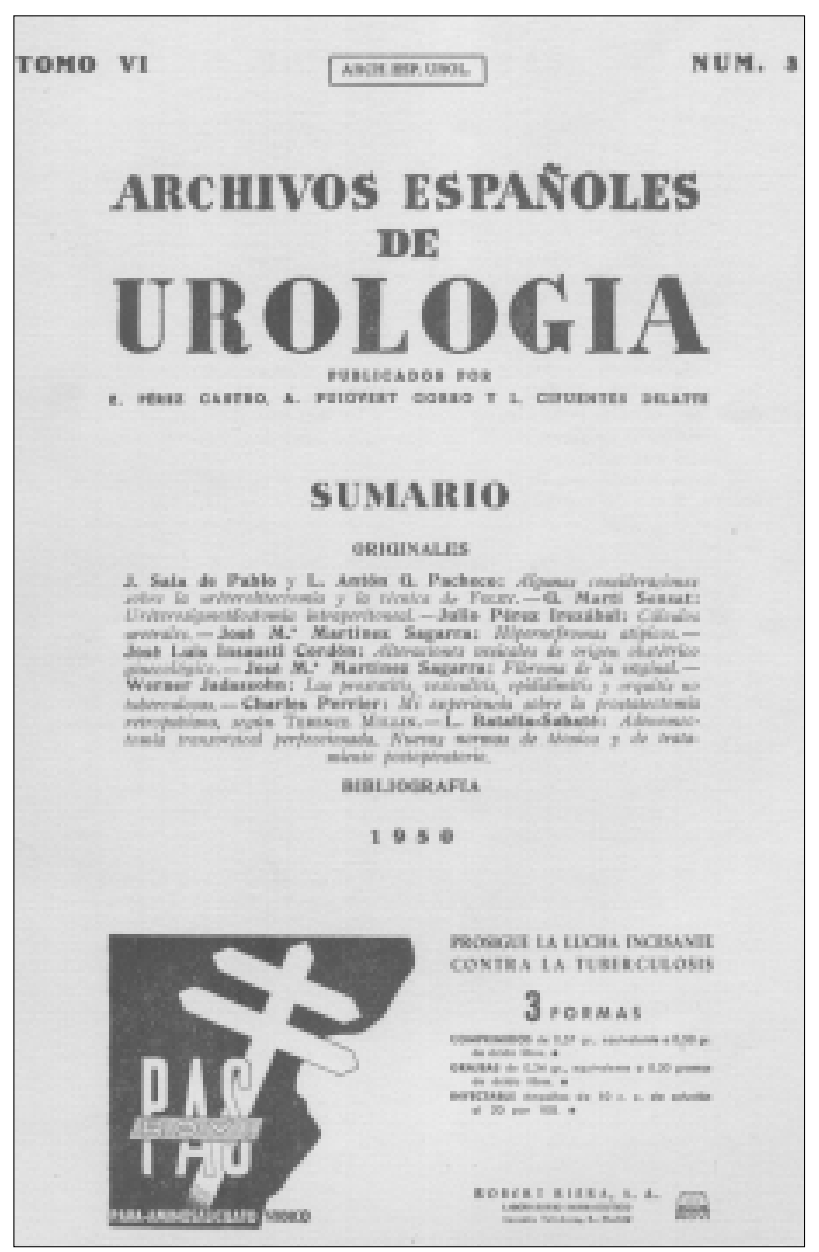

FIGURA 6. Archivos Españoles de Urologia.

Españolas en 1977. Al reiniciarse los Congresos Nacionales, en 1964, recoge en números extraordinarios el contenido de los temas monográficos presentados hasta el año 1967 (entre los años 1960 y 1963 hubo ya temas monográficos pero no he localizado su publicación en revista alguna).

Cirugia Ginecologia y Urologi $a^{20}$, editada en Madrid desde julio de 1950 a 1969, por las figuras de mayor entidad de dichas especialidades, entre los colaboradores sobresalen los Drs. D. Alfonso de la Peña (1904-1971) y D. Salvador Gil Vernet (1892-1987), recoge entre sus páginas bastantes trabajos realizados por urólogos españoles y algunos corresponden a los presentados en los Congresos Nacionales de la AEU, además de algunas noticias sobre ellos (Fig. 7).

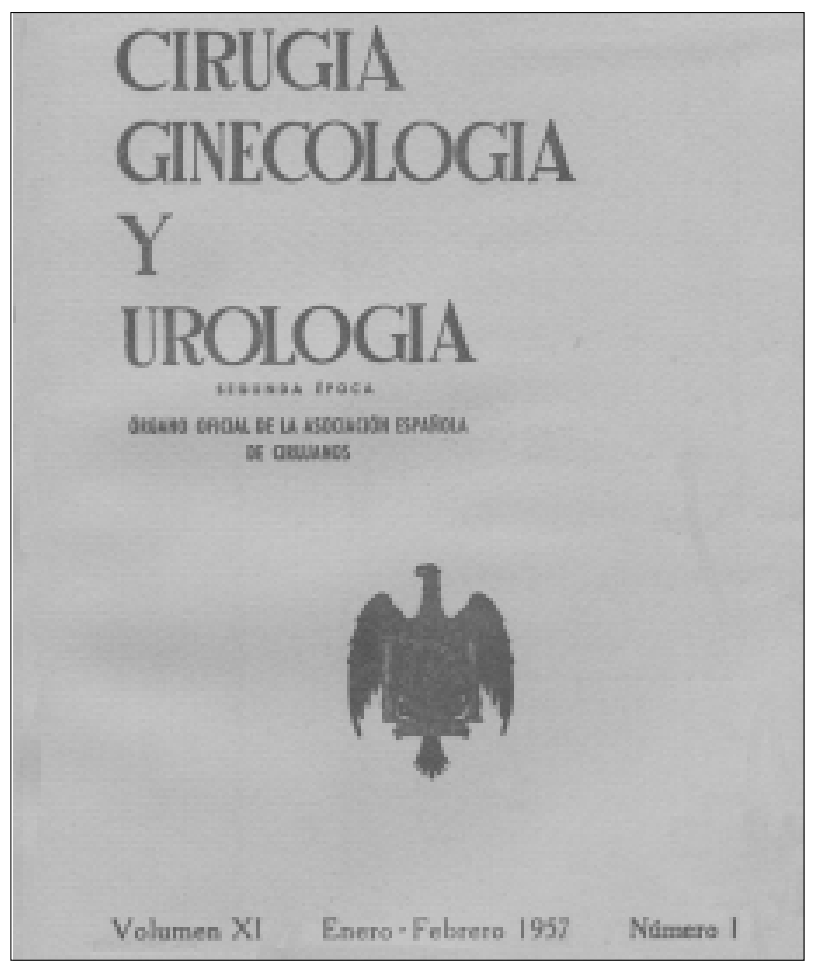

FIGURA 7. Cirugia, Ginecologia y Urología.

Actas de la Asociación Española de Urolo$\boldsymbol{g i a}^{21}$. Tras la celebración del XI Congreso Nacional, en 1946, cesó de un modo radical la actividad científica de la Asociación, hasta que en el año 1952 se reanudó en forma de Reuniones Anuales de la especialidad, en las que únicamente se realizaba la lectura de trabajos para su discusión por los asistentes, de ellas no ha quedado constancia escrita de sus pormenores si exceptuamos el Programa de la Reunión (Fig. 8) entre dicho año y 1967 en el que se recoge el título de las comunicaciones presentadas, conocemos el contenido de algunas de ellas por estar remitidas independientemente por sus autores, para su publicación principalmente en Archivos Españoles de Urología. Desde 1968 con la finalidad de reunir de modo oficial el conjunto de las ponencias y de las comunicaciones de los Congresos de la Asociación, inicia su presentación en Madrid, realizada por la editorial ENE por encargo de la AEU como órgano de difusión las Actas de la Asociación Española de Urología (Fig. 9); de aparición anual continuó su edición hasta 1989 en que cambia de formato y queda como número extraordinario de la Actas Urológicas Españolas. 


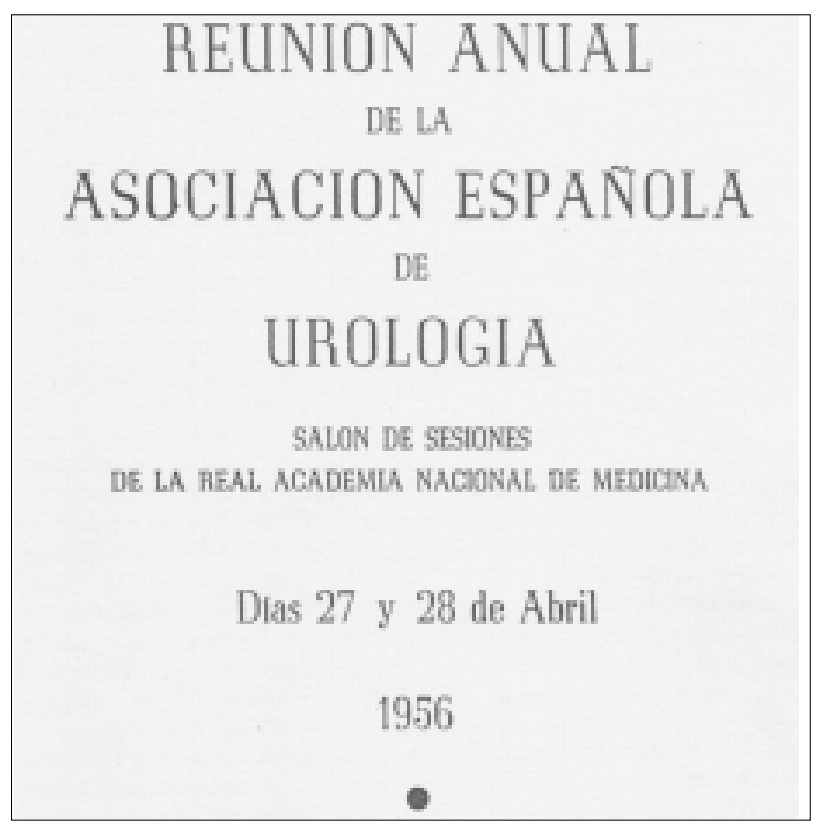

FIGURA 8. Programa de las Reuniones Anuales de la AEU.

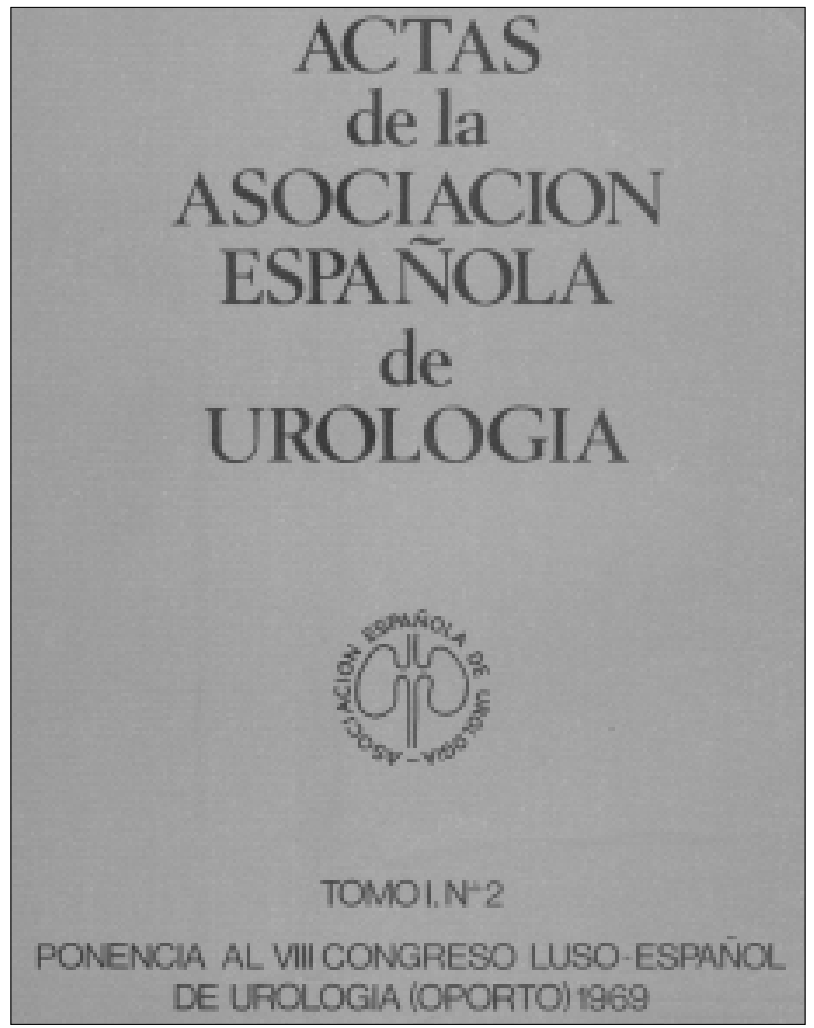

FIGURA 9. Actas de la Asociación Española de Urología.

\section{Reuniones Regionales de la Asociación Es-} pañola de Urología ${ }^{22}$. Revista en la que se recogió, durante quince años, la ingente labor llevada a cabo en las Reuniones Científicas de las Seccio- nes Regionales de la AEU. La editorial Reuniones y Congresos (Reycosa), de la que es propietario D. Luis Guillot, con la colaboración del urólogo Dr. D. Carlos del Río y Rey-Stolle (1909-1983) como director médico, publicó, entre los años 1973 y 1990, gran parte de las comunicaciones presentadas en las reuniones que anualmente organizaban las secciones regionales (excepto de la segunda, correspondiente a Cataluña) a lo que añadió, como números extraordinarios, las ponencias, el fórum y las comunicaciones de varios Congresos Nacionales, los celebrados en León en 1976, en Santander en 1977, en Huelva en 1979, en Acapulco en 1982, en Tenerife en 1985 y en Toledo en 1989; incluye, también, ocasionalmente, noticias de los actos de la AEU, así como alguna nota necrológica, además de recoger trabajos urológicos varios con el título de Comunicaciones urológicas. Con 70 números y en 10 volúmenes, constituye no ya una extraordinaria fuente de información para conocer los trabajos científicos presentados, sino un valioso documento que establece el desarrollo y la evolución de nuestra especialidad, ya que fue testigo del inicio de las Sociedades Autonómicas, además de permitirnos conservar la memoria escrita de casi todos los urólogos españoles de esa época, porque a estas reuniones regionales, casi familiares, la asistencia solía ser masiva y en ellas los jóvenes urólogos se iniciaban en la práctica de exponer sus comunicaciones orales (Fig. 10).

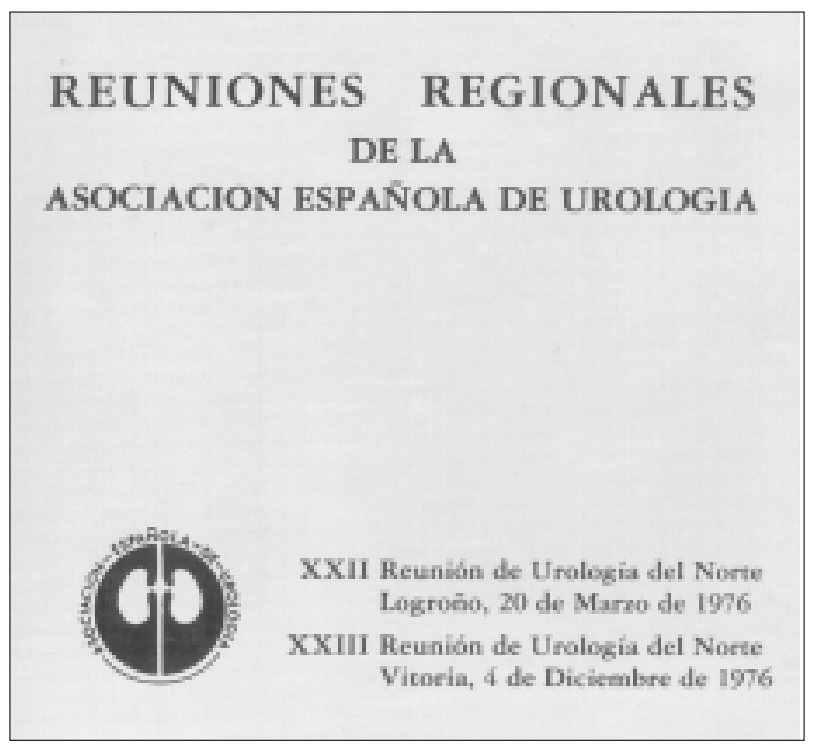

FIGURA 10. Reuniones Regionales de la AEU. 
Circulares Informativas ${ }^{23}$. Entre los años setenta y los ochenta, el secretario de la AEU, ante la falta de otro medio de comunicación directo con los asociados, comienza a remitir con cierta periodicidad circulares en las que da a conocer principalmente las actividades, las sesiones científicas y los Congresos, que se realizaban (Fig. 11). Constituye el precedente que dio origen a la aparición del Boletín Informativo de la AEU en su segunda época.

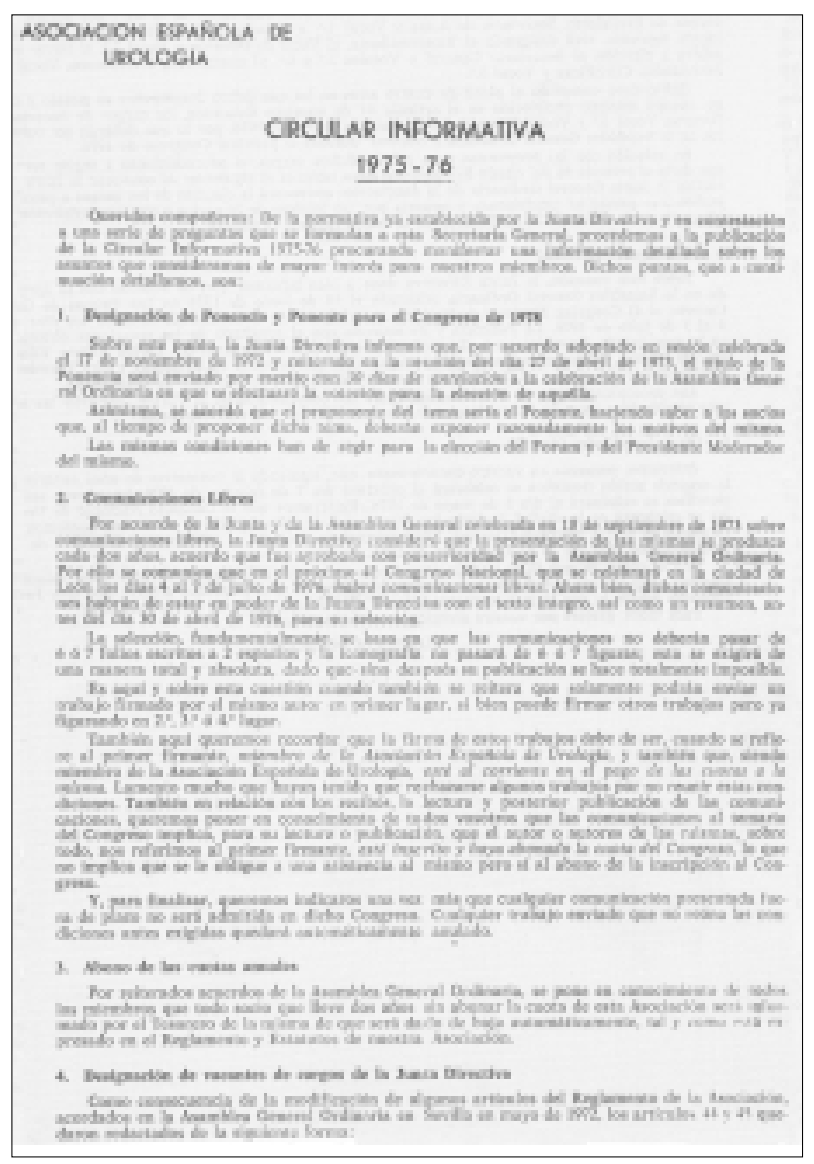

FIGURA 11. Circulares Informativas.

Actas Urológicas Españolas ${ }^{24}$. Por fin en enero de 1977, y por la necesidad de cumplir el mandato estatutario de contar con una publicación propia, inicia su andadura y es hasta la actualidad el órgano representativo oficial de la AEU (Fig. 12); la necesidad de la revista era patente para incrementar los cauces de expresión entre los socios así como la divulgación de sus conocimientos, se concibe como una revista hecha por los urólogos, y para todos los urólogos,

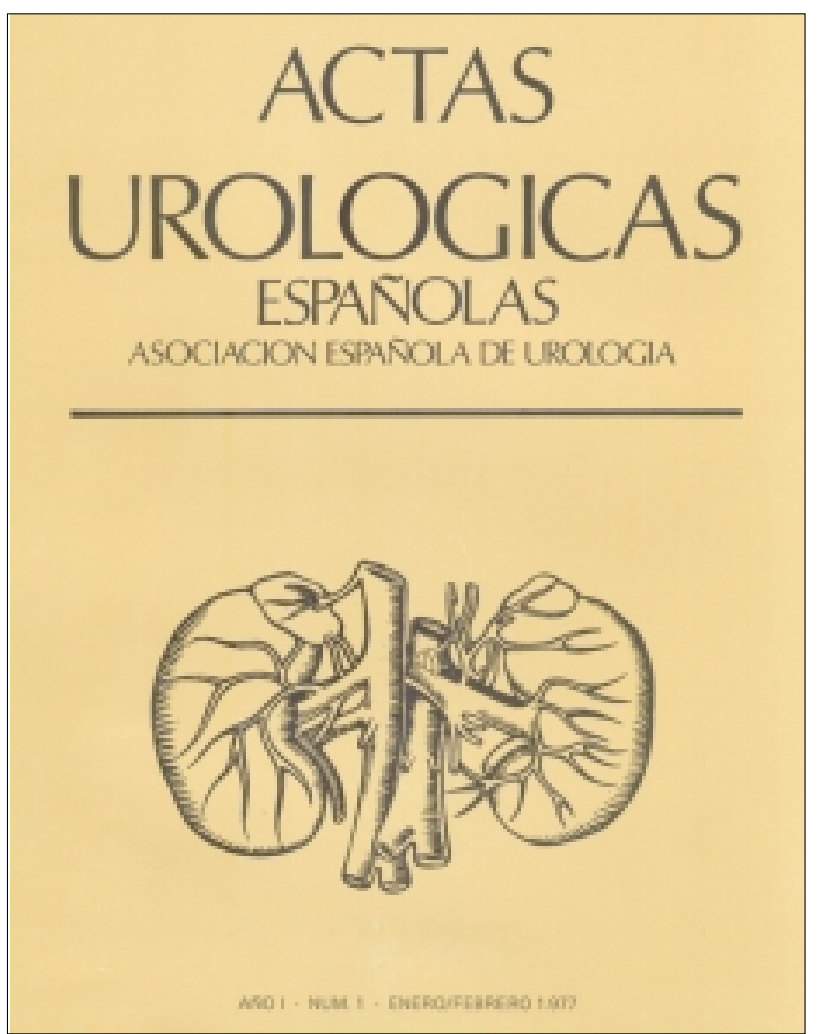

FIGURA 12. Actas Urológicas Españolas.

que sólo puede proporcionar beneficios a la Urología. Su primer director fue el Dr. D. Carlos Alférez Villalobos, quien cumplió la labor de darle estructura científica, mantener su edición y darle prestigio, en 1986 se hace cargo de la dirección el Dr. D. Óscar Leiva Galvis, quien amplió el contenido, modificó el formato inicial y elevó la categoría de la revista, le sucede en enero de 1995 el Dr. D. Joaquín Carballido que logra darle autoridad nacional y una buena consideración internacional, tarea que prosigue con evidente entusiasmo el Dr. D. José Luis Ruiz Cerdá en este 2005.

\section{Boletin Informativo de la Asociación Espa-} ñola de Urologi ${ }^{25}$. Nace en octubre de 1988, con el objetivo fundamental de tener bien informados a los miembros de la Asociación; cuenta como precedente con el Boletín de la Asociación Española de Urología, de 1924, y las Circulares Informativas de los años setenta. La Junta Directiva de la Asociación, consciente de que la comunicación que mantiene con sus socios durante la Asamblea General celebrada anualmente en el Congreso Nacional sobre las activida- 
des realizadas es muy limitada y, en ocasiones, insuficiente, por falta de tiempo para atender todos los puntos de interés tanto administrativos, académicos, docentes, estatutarios, como científicos, crea este Boletín Informativo que distribuye con una frecuencia trimestral o cuatrimestral, y cuyo contenido pasa de tener dos hojas inicialmente hasta contar con 16 páginas en septiembre de 2000 (Fig. 13).

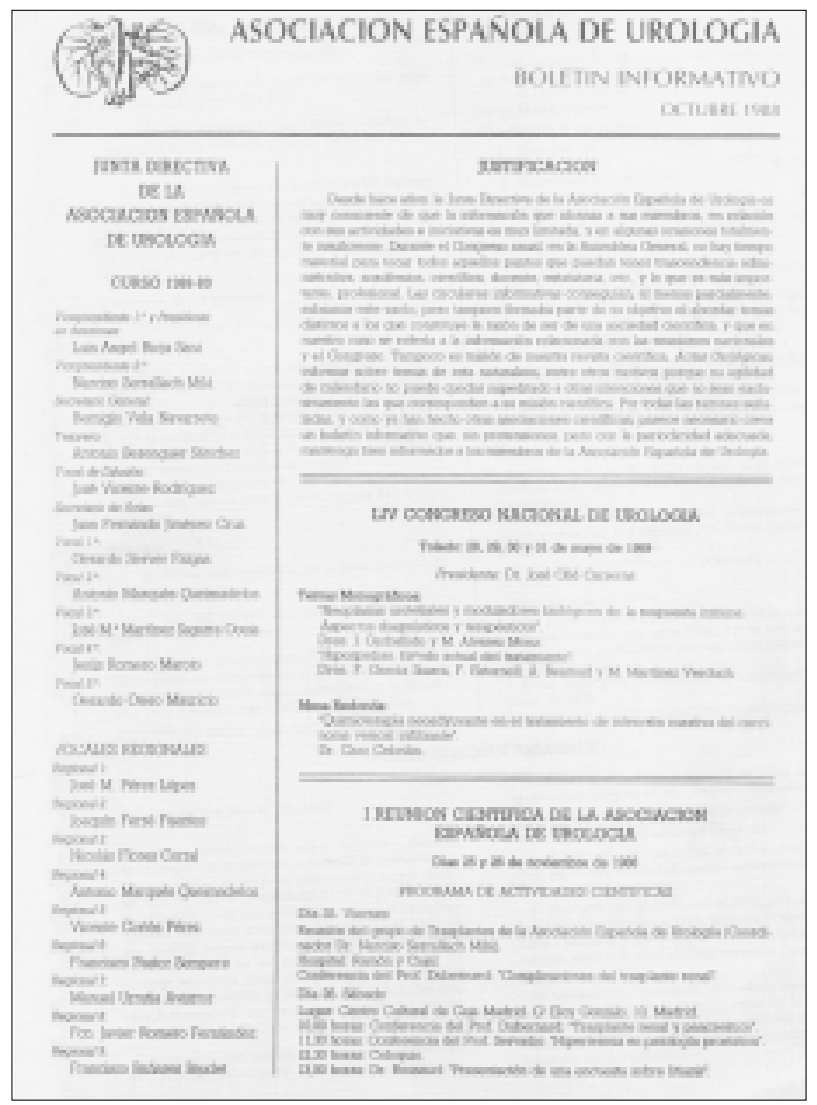

FIGURA 13. Boletin Informativo de la AEU.

En el año 2004, con la entrada del Dr. Carballido como Secretario General de la AEU, de quien depende transmitir la información a los asociados, modifica, el formato del tipo libro inicial al diseño tabloide propio de los periódicos diarios, para exponer, debatir y dar respuesta a aspectos concretos y muy actuales de nuestra especialidad...para intensificar la comunicación dentro y fuera de nuestra Asociación con el enriquecimiento colectivo que de ésto se deriva. Se incrementa el número de páginas a $27 \mathrm{y}$ se incluye, además, datos sobre las actividades, una edi- torial y varias e interesantes secciones con entrevistas, dossier científico, la Urología en los medios de comunicación, etc. (Fig. 14).

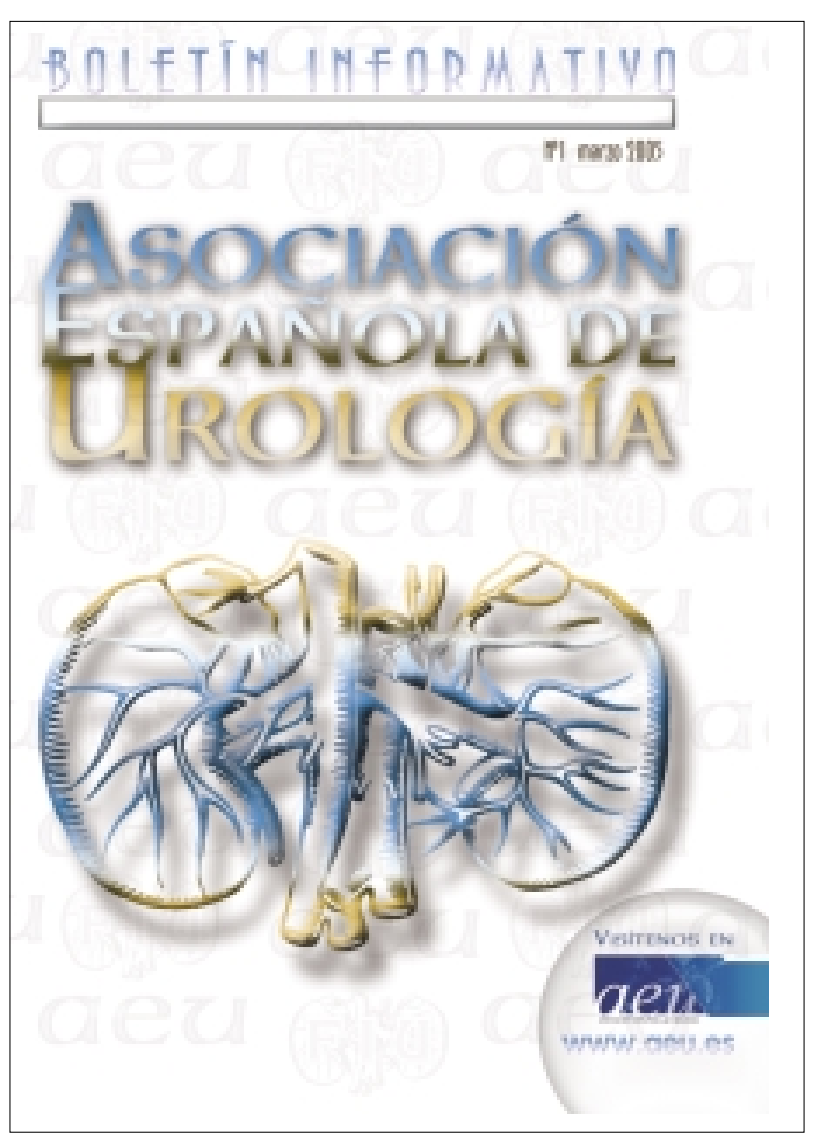

FIGURA 14. Boletín Informativo de la AEU, $2^{a}$ época.

España Médica ${ }^{26}$. Semanario médico, con una aparición tres veces al mes, editado en Madrid desde febrero de 1910, dirigido por el Dr. D. José Elizalde, encontramos entre sus páginas noticias de los Congresos de la AEU, con extracto de las ponencias y relación de las comunicaciones presentadas, además de referencia a las sesiones científicas mensuales y noticias varias relacionadas con sus miembros relatadas con estilo periodístico (Fig. 15).

La Medicina Ibera ${ }^{27}$. Revista semanal de Medicina y Cirugía publicada en Madrid desde 1916 a 1936, en la el urólogo Dr. D. Salvador Pascual Ríos forma parte del comité de redacción responsable de la "Sección de Urología", nos ofrece una información comentada amplia y rigurosa de 
los acontecimientos de la especialidad, además, de que al igual que la publicación anterior, recoger el contenido de los Congresos de Urología y noticias de las actividades llevadas a cabo por la AEU (Fig. 16).

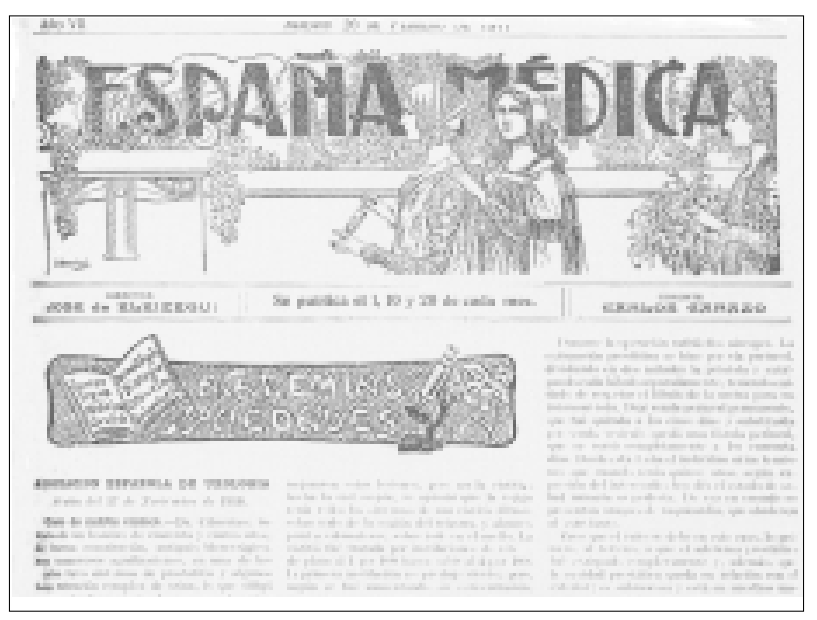

FIGURA 15. España Médica.

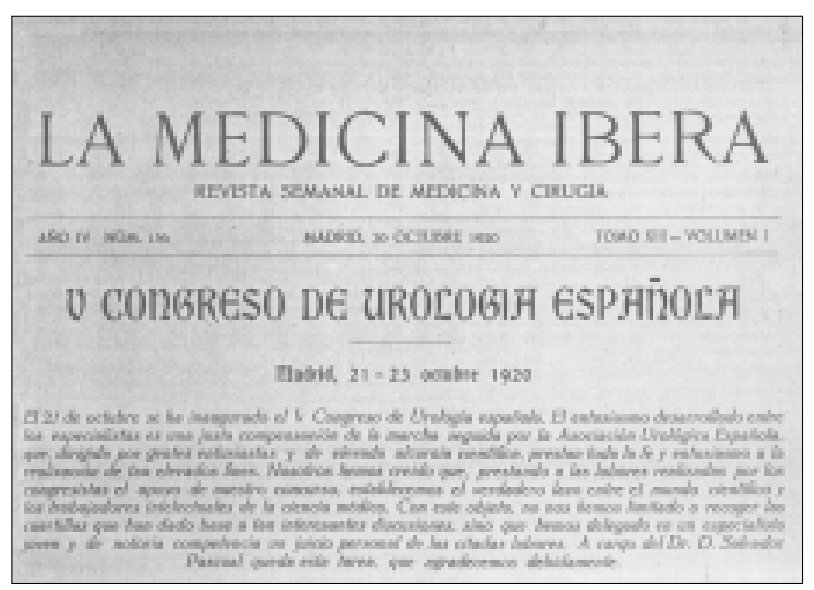

FIGURA 16. La Medicina Ibera.

En cuanto al resto de publicaciones revisadas de esa época como la Revista Clínica de Urología y Cirugía Génito-Urinaria, editada en Madrid, en enero de 1914 y dirigida por el Dr. D. Rafael Mollá y Rodrigo (1862-1930) de aparición bimestral, tuvo dos años de duración y en ella se recoge únicamente el contenido de la primera sesión mensual de la AEU, por lo que al igual que en la valenciana Policlínica y la murciana Murcia Médica, dados los pocos trabajos recogidos en ellas así como la escasa información que se relaciona con la AEU nos hace no incluirlas en esta relación. Del resto de las revisadas como El Siglo
Médico, Anales del Instituto Rubio, Revista Clínica de Madrid, Revista Clinica Española, Los Progresos de la Clínica, todas ellas de Madrid, ciudad donde tenía lugar la actividad científica, no hemos encontramos referencias ni noticias de relevancia sobre la Asociación.

\section{DISCUSIÓN}

Como vemos cuatro publicaciones han ostentado el título de órgano oficial de la Asociación desde 1924 hasta que, en 1977, se edita Actas Urológicas Españolas (Tabla 1). Las actividades llevadas a cabo por los miembros de la Asociación han quedado recogidas en doce publicaciones diferentes, cinco de ellas contemplan el contenido de los Congresos Nacionales (Tabla 2), otras cinco recogen las actividades periódicas (Tabla 3), y las otras dos corresponden a semanarios médicos de la época que con estilo periodístico informan de los diferentes actos y resumen su conte-

Tabla 1.

Publicaciones que han llevado el título de Órgano Oficial de la AEU

Revista Española de Cirugía

y Urología

enero1924-junio1933

Revista Española de Urología julio 1933-junio 1936

Archivos Españoles de Urología junio 1964-enero1977

Actas Urológicas Españolas enero 1977-2006

\section{Tabla 2}

Publicaciones que recogen el contenido de los Congresos Nacionales de Urología

1911-1946 Actas de los Congresos Nacionales de la AEU 1964-1967 Archivos Españoles de Urología

1969-1989 Actas de la Asociación Española de Urología 1976-1979 Reuniones Regionales de la AEU

1989-2000 Actas Urológicas Españolas

\section{Tabla 3}

Actividades científicas de la AEU

1915-1924 Revista Española de Urología y Dermatología

1924-1933 Revista Española de Cirugía y Urología

1933-1936 Revista Española de Cirugía

1945-1977 Archivos Españoles de Urología

1977-2006 Actas Urológicas Españolas 
nido; por último el Secretario General de la Asociación ha dispuesto en este periodo de cuatro diferentes medios de comunicación para dar información a los asociados (Tabla 4).

\section{Tabla 4}

Medios Informativos utilizados por el Secretario de la AEU

\begin{tabular}{ll}
\hline $1923-1924$ & Boletín de la AEU \\
$1952-1967$ & Programa de las Reuniones Anuales \\
$1970-1988$ & Circular Informativa \\
$1988-2004$ & Boletín Informativo 1a época \\
2004 & Boletín Informativo 2ª época \\
\hline
\end{tabular}

\section{CONCLUSIONES}

Las actividades llevadas a cabo por la Asociación Española de Urología a lo largo de sus noventa y cinco años de existencia han quedado recogidas en las publicaciones periódicas de la época, la existencia de todas estas revistas y el conocimiento de su contenido nos permiten conocer y valorar la labor y el trabajo realizado por sus miembros, así como acercarnos al de las personas que, poco a poco, han contribuido a hacer que la Urología española alcance y mantenga el nivel que le corresponde en el contexto urológico internacional.

\section{REFERENCIAS}

1. Pérez Albacete, M. Congresos celebrados por la Asociación Española de Urología en el siglo XX, Urol. Integr. Invest. 2002;7(2):205-212.

2. Insausti Cordón, J. L. Compendio histórico de la Urología Española y de su Asociación". Ponencia IV Congreso Iberoamericano de Urología, Acapulco, 1982.

3. Maganto Pavón, E. Historia del periodismo urológico en España I-II-III. Arch Esp Urol 1996;49(9):889-922.

4. Primera Reunión de la Asociación Española de Urología Temas oficiales, comunicaciones y discusiones. Impr. Casa Vidal, Madrid, 1912.
5. Segunda Reunión de la Asociación Española de Urología. Temas oficiales, comunicaciones y discusiones. Impr. Casa Vidal, Madrid, 1915.

6. IV Congreso Hispano-Portugués de Urología. Libro de Actas. Impr. Ministerio de Marina, Cádiz, 1935

7. Pérez Albacete, M. Fundación y Desarrollo de la Asociación Española de Urología, en Historia Biográfica y Bibliográfica de la Urología Española en el siglo XX, pág. 61-63, Edicomplet, Madrid, 2002.

8. V Congreso Hispano-Portugués de Urología. Libro de Actas. Impr. Ministerio de Marina, Madrid, 1947.

9. Arch Esp Urol 1964, XVII (1).

10. Acta de la Junta General de la Reunión de la Asociación Española de Urología, Málaga, 2 de junio de 1968.

11. Acta de la Junta General Extraordinaria de la Reunión de la Asociación Española de Urología, Oporto, 25 de junio de 1969.

12. Acta de la Junta Directiva de la Asociación Española de Urología, Madrid,12 de noviembre de 1976.

13. Nuevo reglamento y directorio. Pág. 11 y 25. Actas Urológicas Españolas Ene Edic. Madrid, 1998

14. Actas de los Congresos de la Asociación Española de Urología. Temas Oficiales, comunicaciones y discusiones. (19121947). Madrid, Lisboa.

15. Revista Española de Urología y Dermatología. (1914-1924), Madrid.

16. Revista Española de Cirugía y Urología. (1924-1933), Madrid.

17. Boletín de la Asociación Española de Urología. (1923-1924).

18. Revista Española de Urología. (1933-1936), Madrid.

19. Archivos Españoles de Urología. (1944-2005), Madrid.

20. Cirugia, Ginecologia y Urologia. (1950-1969), Madrid.

21. Actas de la Asociación Española de Urología. (1968-1979), Madrid.

22. Reuniones Regionales de la Asociación Española de Urología. Reycosa, (1975-1990), Madrid.

23. Circulares Informativas. (1975-1988), Madrid.

24. Actas Urológicas Españolas. (1977-2006), Madrid.

25. Boletín Informativo de la Asociación Española de Urología. (1988-2006), Madrid.

26. España Médica. (1910-1936), Madrid.

27. La Medicina Ibera. (1916-1936), Madrid.

Dr. M. Pérez Albacete

Email: mariano.perez2@carm.es

(Trabajo recibido el 19 de diciembre 2005) 